$54^{\text {ème }}$ Congrès de la SFMBCB, 03003 (2011)

DOI: $10.1051 / \mathrm{sfmbcb} / 20115403003$

(C) Owned by the authors, published by EDP Sciences, 2011

\title{
Lymphome malin non hodgkinien mandibulaire primitif : à propos d'un cas
}

\author{
Tabeti-Bentahar $\mathbf{C F}^{\mathbf{1}}$, Benayada $\mathbf{L}^{\mathbf{1}}$, Bouzouina $\mathrm{F}^{\mathbf{1}}$, Bénali $\mathrm{F}^{\mathbf{2}}$ \\ ${ }^{1}$ Service de Pathologie et Chirurgie buccale, CHU, Oran, Algérie \\ ${ }^{2}$ Anatomopathologiste libéral, Oran, Algérie \\ tabeti_cherifa@yahoo.fr
}

Pour les lymphomes malins non hodkinien (LMNH), la nouvelle classification de l'Organisation Mondiale de la Santé de 2001 définit une quarantaine d'entités. Les LMNH sont des proliférations malignes intéressant le tissu lymphoïde et ils représentent la majorité des lymphomes. Le LMNH représente l'hémopathie maligne la plus fréquente avec un taux annuel standardisé à la population mondiale de 6,7 pour 100000 habitants par an. Selon Haioun et al. (2002), 60\% des LMNH correspondent à l'entité histopathologique « diffus à grandes cellules B » qui constitue l'exemple de lymphome dit «agressif », caractérisé à la fois par une évolution spontanée rapidement fatale mais aussi par une possibilité de guérison après traitement. Un cas de LMNH mandibulaire est apporté. Il s'agit d'un homme âgé de 41 ans, mécanicien, fumeur, sans antécédents médicochirurgicaux particuliers, qui a été adressé en juin 2007 par un confrère chirurgien dentiste pour hypoesthésie labio-mentonnière gauche et des douleurs hémifaciales homolatérales, rebelles aux antalgiques, apparues après à l'extraction de la 36. L'état général était bon. L'examen clinique a retrouvé une légère tuméfaction de la branche horizontale gauche de la mandibule faisant corps avec l'os et un signe de Vincent positif (hypoesthésie cutanéomuqueuse). La radiographie panoramique a révélé une radiotransparence, le CT-scan une déminéralisation osseuse, se rehaussant modérément après injection du produit de contraste. La biopsie a orienté vers un lymphome ; l'immunohistochimie a permis de poser le diagnostic de lymphome à grandes cellules B (positivité au marquage par l'anticorps anti-CD 20). Le patient a été confié au Service d'Hématologie, puis il a été perdu de vue. En octobre 2007, le patient a été revu avec une extension locorégionale : la tuméfaction jugale mesurant $6,5 \times 5,5 \mathrm{~cm}$. L'examen endobuccal a retrouvé une masse de $3 \mathrm{~cm}$ dans son grand axe avec extension du côté vestibulaire et du côté lingual. Le CT-scan a montré une masse tumorale avec envahissement des tissus mous et de la région parapharyngée. Le patient a été réadressé dans le Service d'Hématologie. Un bilan d'extension a été réalisé (téléthorax, échographie abdominopelvienne, biopsie médullaire, dosage de la lacticodeshydrogénase ; tous ces examens étaient normaux) a permis de classer le patient au stade II selon la classification d'Ann Arbor. Le patient a été mis sous protocole CHOP ( Doxorubicine $50 \mathrm{mg} \cdot \mathrm{m}^{-2}$, Cyclophosphamide $750 \mathrm{mg} . \mathrm{m}^{-2}$, Vincristine $1,4 \mathrm{mg} \cdot \mathrm{m}^{-2}$, Prednisone $40 \mathrm{mg} / \mathrm{m}^{-2}$ de $\mathrm{J} 1$ à $\mathrm{J} 5$.).Dès la 1ère cure, il y a eu régression totale de la tuméfaction; seule l'hypoesthésie labiale inférieure persistait. A ce jour, onze cures ont été faites ; la radiothérapie n'a pas été indiquée. Actuellement, le patient se porte bien.

La localisation mandibulaire des LMNH est exceptionnelle (Bachaud et al. 1992). Le LMNH survient à tout âge avec un pic de fréquence vers 46 ans (Saint Blancard et al. 2001) et une légère prédominance masculine : sex ratio de 1,5 (Ahossi et al. 2006). Les manifestations révélatrices ne sont pas spécifiques et peuvent simuler une ostéite ou une tumeur bénigne des maxillaires. Les signes radiologiques sont pauvres: ostéolyse mal délimitée, parfois ostéocondensation périphérique. L'étiopathogénie des LMNH n'est pas encore élucidée. Le diagnostic des LMNH repose sur l'examen anatomopathologique complété par un examen immunohistochimique. Le bilan d'extension locorégionale et générale permet la classification des LMNH selon la classification d'Ann Arbor. Le traitement des LMNH repose sur l'association d'une polychimiothérapie (CHOP ; R-CHOP ; CHOP-bléo; M Bacod...) et de la radiothérapie. Le pronostic des LMNH repose sur l'Index Pronostique International (IPI).

This is an Open Access article distributed under the terms of the Creative Commons Attribution-Noncommercial License 3.0, which permits unrestricted use, distribution, and reproduction in any noncommercial medium, provided the original work is properly cited. 